\title{
INVESTIGATION OF NEWTONIAN AND NON-NEWTONIAN DROPLETS IN COLLISION WITH THE HEATED ULTRAPHOBIC SURFACE ON VARIOUS WEBER NUMBERS
}

\author{
Morteza Bayareh \\ Keywords: Hydrophobic surfaces, VOF method, Weber number, Newtonian Droplet, Non-Newtonian \\ droplets
}

\begin{abstract}
Super hydrophobic surfaces due to water repelling property, have a considerable potential for the use in various modern technologies. In this paper, the collision between Newtonian and non-Newtonian droplets with a superhydrophobic hot surface like graphite has been simulated. The contact angle is 140 and 160 degree. Simulation yielded proper result compared to the experimental results. It is found that the temperature effect on hydrophobic surfaces is negligible. Our results demonstrate that the dimensionless drop diameter increases with the Weber number. It is revealed that a Newtonian droplets shows different behavior compared to a non-Newtonian one especially at higher temperatures.
\end{abstract}

\section{INTRODUCTION}

One of the most important researches that have attracted a lot of attention in recent years, reducing the drag of flow on hydrophobic surfaces is covered. The drag reduction of Newtonian fluids in pipes with repellent walls was studied by Watanabe and Udagawa[1]. Experiments were carried out to measure the pressure drop and the velocity profile of tap water and an aqueous solution of glycerin flowing in pipes with highly water-repellent walls, by using a pressure transducer and a hot-film anemometer, respectively. The same drag reduction phenomena also occurred in degassed tap water when using a vacuum tank. The velocity profile measured in this experiment gives the slip velocity at the pipe wall, and it was shown that the shear stress was directly proportional to the slip velocity. Tretheway and Meinhart[2] measured fluid motion through a 30×300 micron channel by micron-resolution particle image velocimetry. They showed the average velocity profiles for flow near hydrophilic (squares) and hydrophobic (triangles) microchannel surfaces. For flow through a hydrophobic microchannel, the velocity profile was significantly different. Effects of hydrophobic surface on skin-friction drag were investigated through direct numerical simulations of a turbulent channel flow by Kim et al [3]. It was found that near-wall turbulence structures were modified differently, resulting in drag increase. It was also found that the slip length must be greater than a certain value in order to have a noticeable effect on turbulence. An important implication of the present finding was that drag reduction in turbulent boundary layers was unlikely with hydrophobic surface with its slip length on the order of a submicron scale. Choi et al [4] examined the slip effects of water flow in hydrophilic and hydrophobic microchannels of 1 and $2 \mu \mathrm{m}$ depth. The flow rates of pure water at various applied pressure differences for each surface condition were measured using a high-precision flow metering system and compared to a theoretical model that allows for a slip velocity at the solid surface. The slip length was found to vary approximately linearly with the shear rate. A series of experiments was presented which demonstrate significant drag reduction for the laminar flow of water through microchannels using hydrophobic surfaces with well-defined micron-sized surface roughness by Perot and $\mathrm{Ou}$ [5]. An experimental flow cell was used to measure the pressure drop as a function of the flow rate for a series of microchannel geometries and ultrahydrophobic surface designs. Pressure drop reductions up to $40 \%$ and apparent slip lengths larger than $20 \mu \mathrm{m}$ were obtained using ultrahydrophobic surfaces. No drag reduction was observed for smooth hydrophobic surfaces. YU Yung Sheng et al [6] studied the physical mechanism of the drag reduction of hydrophobic materials in the macroscopic scale. The experiment included the drag and velocity measurements of laminar boundary layer flow over flat plates, and the observation of air bubbles on the surface. In the drag measurements, the plates with bubbles on the surface leaded to drag reduction, but not for those without bubbles. Velocity measurement confirmed that the flow was laminar and gived apparent fluid slip on the plate wall with bubbles. In observation, air bubbles in macroscopic size emerged and enlarged on This paper was recommended for publication in revised form by Regional Editor Kwok-wing Chau Assistant Professor, Faculty of engineering, Shahrekord University, Shahrekord, Iran E-mail address: m.bayareh@eng.sku.ac.ir 
hydrophobic surfaces but not on hydrophilic surfaces. Daniello et al [7] examined the drag reduction of turbulent flow in superhydrophobic surfaces. Particle image velocimetry and pressure drop measurements were used to observe significant slip velocities, shear stress, and pressure drop reductions corresponding to drag reductions approaching 50\%. At a given Reynolds number, drag reduction was found to increase with increasing feature size and spacing, as in laminar flows. Pittoni et al [8]studied on water drops impingement onto two different graphite substrates. They measured Wetting diameter, dynamic contact angle, and drop shapes. Phenomenology and causes of the superhydrophobicity failure were investigated. Droplet impingement experiments at low Weber numbers were conducted by digitizing silhouettes of impacting water drops onto unlike graphite substrates, typified by different advancing water contact angles $(\theta a)$ : 140 and $160^{\circ}$. The purpose was to carefully investigate the phenomenology and possible causes of the failure of the superhydrophobicity. During impact and spreading phases, all the drops impinging onto both graphite substrates showed a similar behavior. In this study, we investigate the numerical treatment of water with hydrophobic surface. Our model is based on Volume of Fluid (VOF) method.

\section{GOVERNING EQUATIONS}

One of the most important parameters in the study of solid liquid interface is the difference between the contact angle and roughness of a surface. The contact angle is due to roughness and non-uniformity of the surface. Thus, the difference between the contact angle and the roughness of a surface is established as follows:

$\theta_{a d v}-\theta_{r e c}=\left(1-f_{L A}\right) R_{f} \frac{\cos \theta_{a 0}-\cos \theta_{r 0}}{-\sin \theta}=\left(\sqrt{1-f_{L A}}\right) R_{f} \frac{\cos \theta_{r 0}-\cos \theta_{a 0}}{\sqrt{2\left(R_{f} \cos \theta_{0}+1\right)}}$

For the homogeneous interface $\mathrm{f}_{\mathrm{LA}}=0$. In contrast, in non-homogeneous interface $\mathrm{f}_{\mathrm{LA}}$ will be non-zero value.

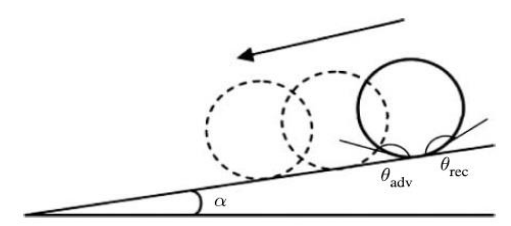

Figure. 1. The liqiud drop on a ramp.

The motion for the free surface is governed by Navier-Stokes equations. If the flow is considered incompressible, the Navier-Stokes equation becomes:

$\mathrm{R} \frac{\partial \boldsymbol{V}}{\partial t}+\nabla \cdot(\boldsymbol{V} \boldsymbol{V})=-\frac{1}{\rho} \nabla P+\frac{1}{\rho} \nabla \cdot \boldsymbol{\tau}+\boldsymbol{g}+\frac{1}{\rho} \boldsymbol{F}_{b}$

This equation is valid for the whole flow field, even if the density field, $\rho$, and the viscosity field, $\mu$, change discontinuously. Here Vis the fluid velocity, $p$ is the pressure, and $g$ is the acceleration due to gravity. $\tau$ is the shear stress tensor. $\mathbf{F}_{\mathbf{b}}$ is the forces over the entire surface. The integral is over the interface between the two fluids. The fluids are incompressible. The continuity equation is given as follows:

$$
\nabla . \mathbf{u}=0
$$

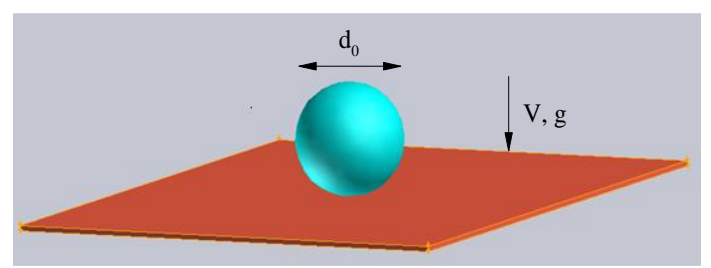

Figure. 2. The geometry of collision with the hydrophobic surface. 


\section{RESULTS AND DISCUSSION}

In this section, we study the dependence of fluid properties such as viscosity, density and surface tension on temperature and obtaining the relationship among these parameters and temperature in order to use their coefficients on Fluent software.

All properties have been extracted after comparison the results and diagrams depending on the input type on Fluent software.

$$
\begin{aligned}
& \sigma=-168 \times 10^{-6} T+122 \times 10^{3} \\
& \rho=-3.236 \times 10^{-3} T^{2}+1.65 T+792.35 \\
& \mu=6.5 \times 10^{9} T^{-5.2}
\end{aligned}
$$

the numbers in the above relations is placed in the section of "Material", instead of default numbers on fluent software.

Because of the symmetry, we use axisymmetric mode and axis definition in this problem. To create drop, we must use marking it and Patching.

Before results presentation, it is necessary to check the Solution Accuracy Independence on the number of network nodes. It can be seen that by increasing the number of elements, Results Variations reduce and the solution goes to a specific value. In this case, if the number of elements is increased to more than 80000 in 2D solving and more than 70000 to 3D solving, the response will be independent of the number of elements.

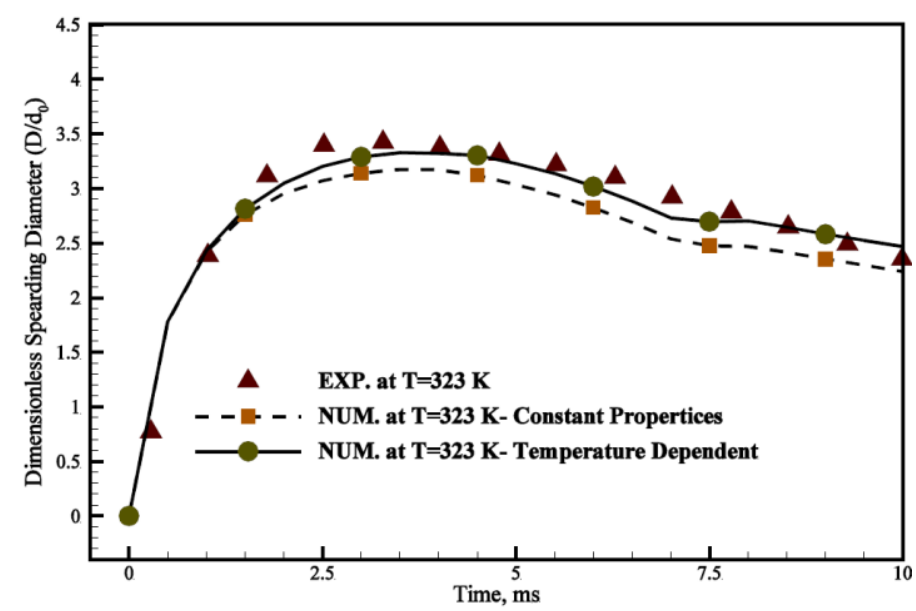

Figure. 3. Dependence on accuracy of solutions by using temperature dependent properties.

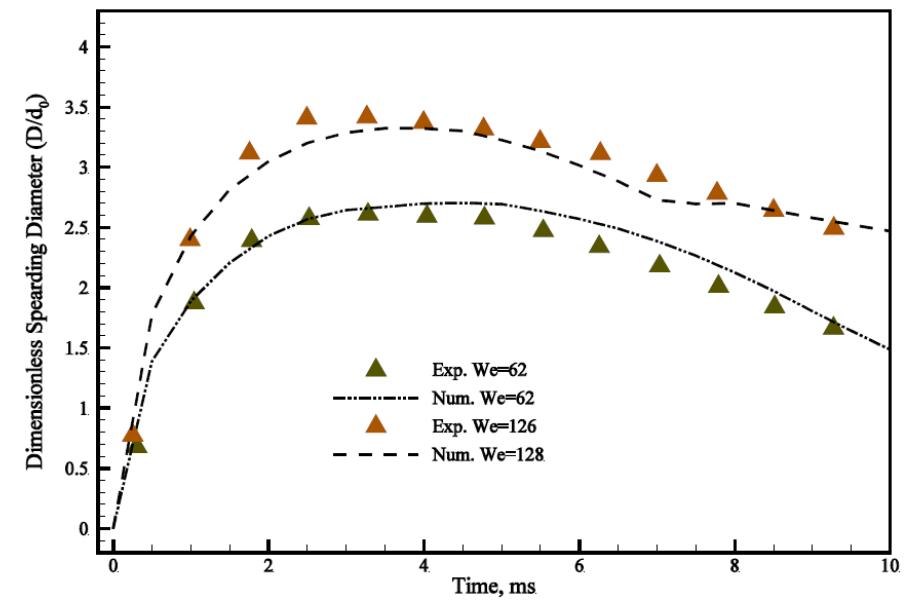

Figure. 4. Distribution drops Newtonian behavior in different Weber numbers. 
Figure 3 shows the dimensionless drop diameter for three different experimental situations, temperatureindependent properties and temperature-dependent properties for a Newtonian fluid. As you can see, when using the fitted relationship, the calculations accuracy is increasing extremely and the results have fewer errors than the temperature-independent properties mode. Thus, the present work is based on the properties dependence on the temperature along the process.

In addition, validation of the project is done based on the data and diagram which is presented in reference 35 and can be realized that the used method validate for simulation.

Figures 4 and 5 is showing the comparison of results of the diameter changing simulation over time in the present work and experimental data for both Newtonian and non-Newtonian fluids in different Weber numbers such as 64 and 126.

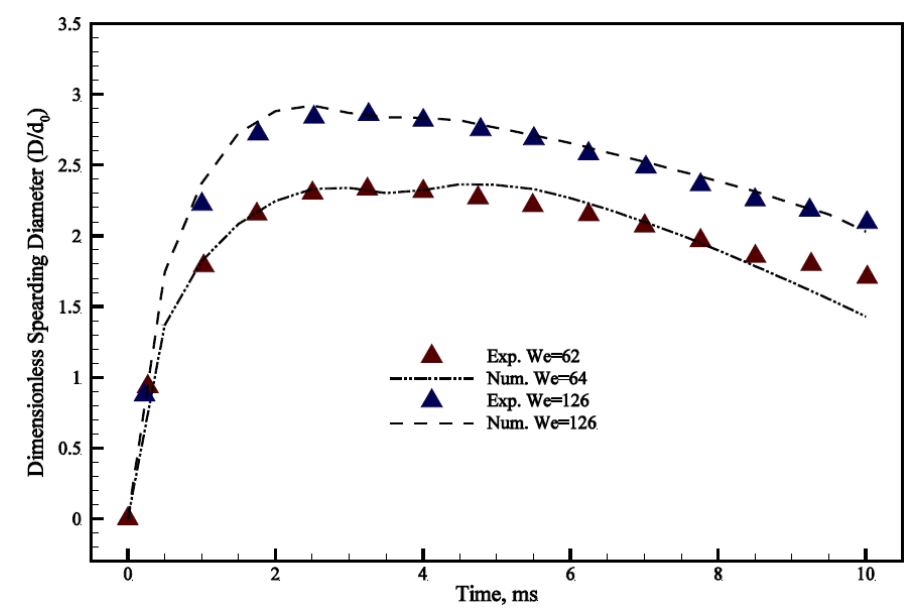

Figure. 5. Distribution of non-Newtonian behavior of the drop in the numbers of different Weber.

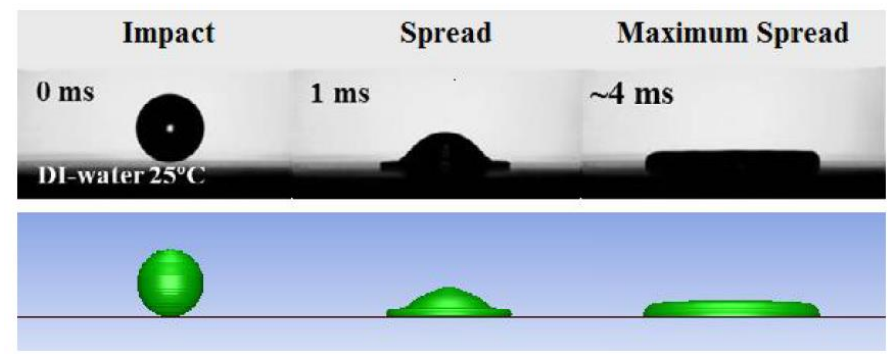

Figure. 6. Visual comparison of experimental results with simulation for Weber number 62 (Newtonian fluids)

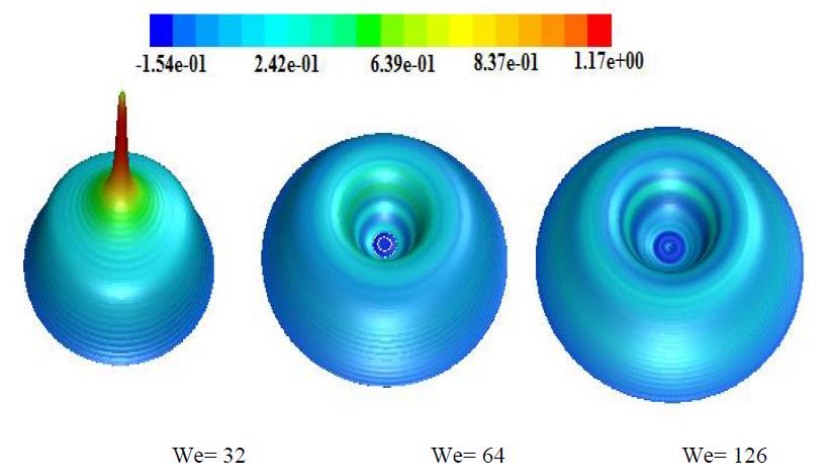

Figure. 7. Non-Newtonian radial velocity drops during $8 \mathrm{~ms}$. 
According to the diagrams leading, it is obvious that at first the wet diameter has increased and then has decreased. Also figure 6 is showing the comparison for three situations of hitting, spreading and maximum diameter at 0,1 , and $4 \mathrm{~ms}$ for Newtonian fluids in Weber number 62.

Figure 7 is showing the radial velocity for non-Newtonian drop in Weber numbers 32, 63 and 126 at 8 ms. These figures are determining that the maximum speed in drop tower occurs when the Weber number is 32 .

Figure 8 is showing the results for different Weber numbers and determines that the difference between Newtonian and non-Newtonian fluid behavior is more sensible by increasing the Weber number. Also figures 9 and 10 is showing the comparison between the results of temperature effect on the behavior of Newtonian and non-Newtonian droplets for different weber numbers in hydrophilic surface.

Figures 11 and 12 show the surface temperature effects with different contact angles on drop behavior for Newtonian and non-Newtonian drops respectively. As it's known, by increasing the contact angle from 80 as a hydrophilic surface to the contact angle of 150 as a super-hydrophobic surface, the effects of temperature on drop behavior becomes less for both Newtonian and non-Newtonian fluids.

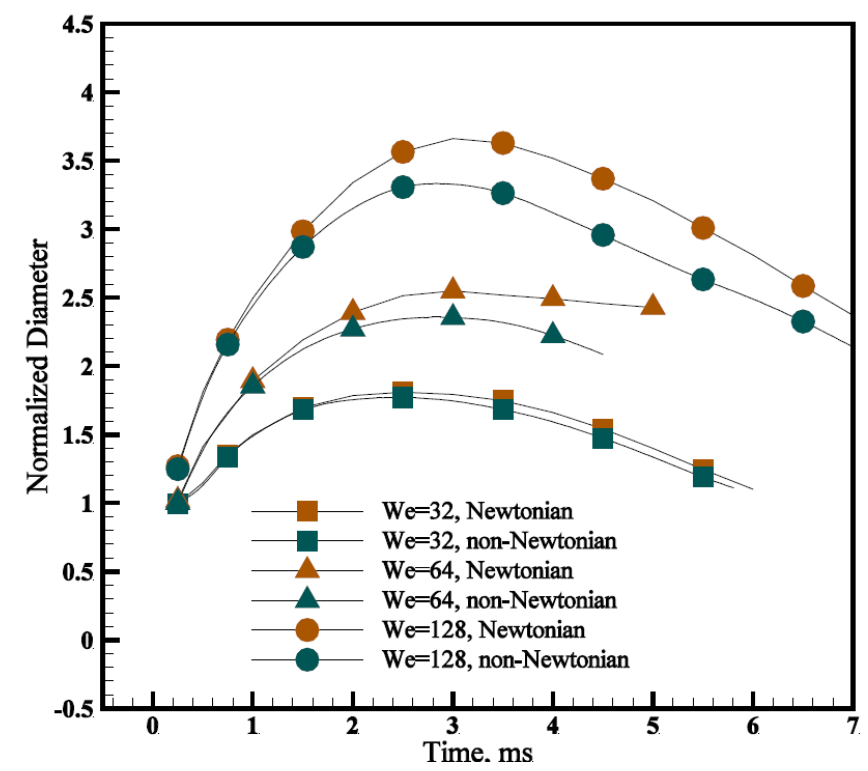

Figure. 8. Drop diameter sensitivity to be Newtonian and non-Newtonian in the different Weber numbers

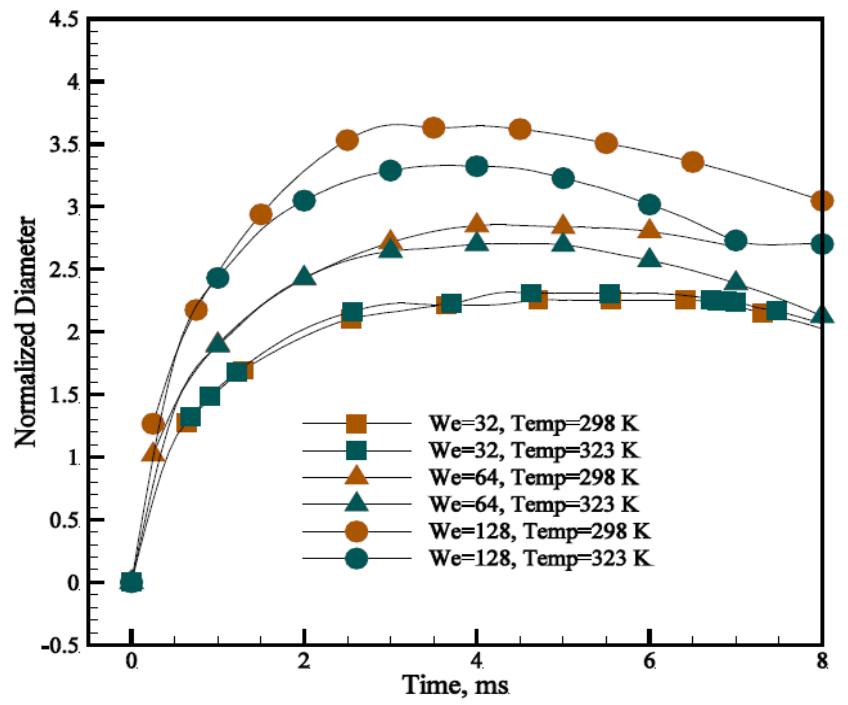

Figure. 9. Temperature effects of the Newtonian droplets behavior in different Weber numbers for the hydrophilic surface 


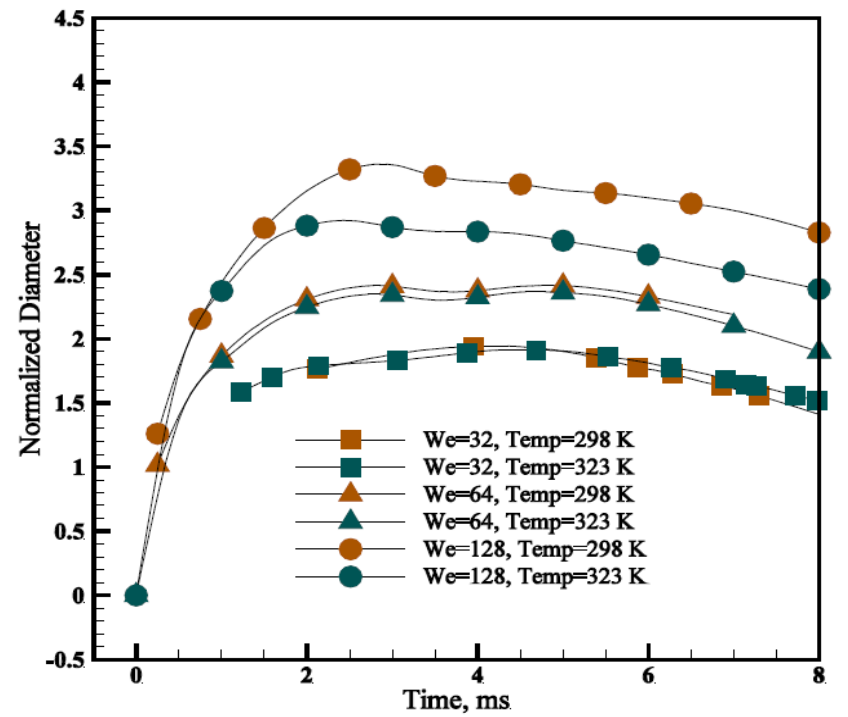

Figure. 10. Temperature effects of the non-Newtonian droplets behavior in different Weber numbers for the hydrophilic surface.

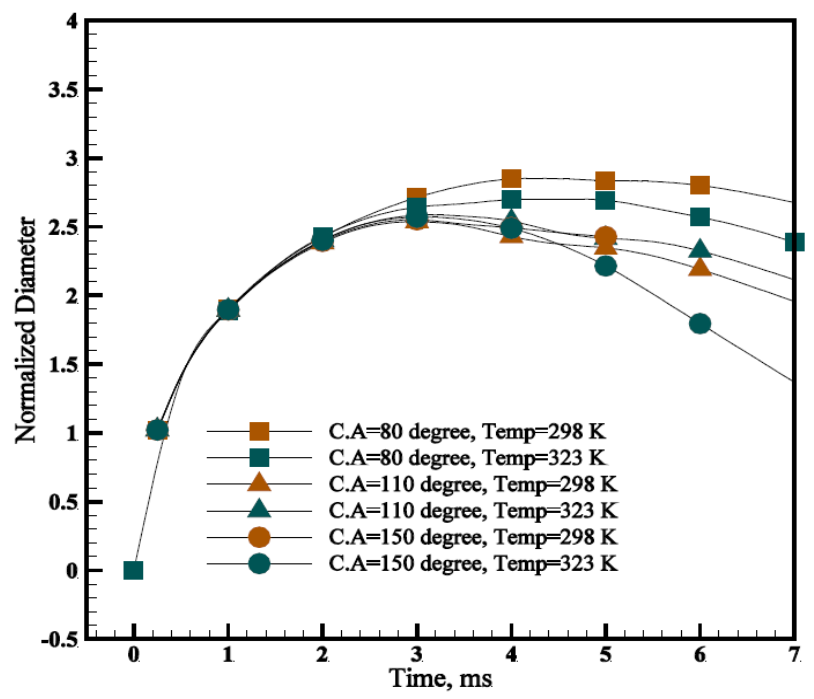

Figure. 11. Temperature effects of Newtonian droplet behavior of surface with different contact angles

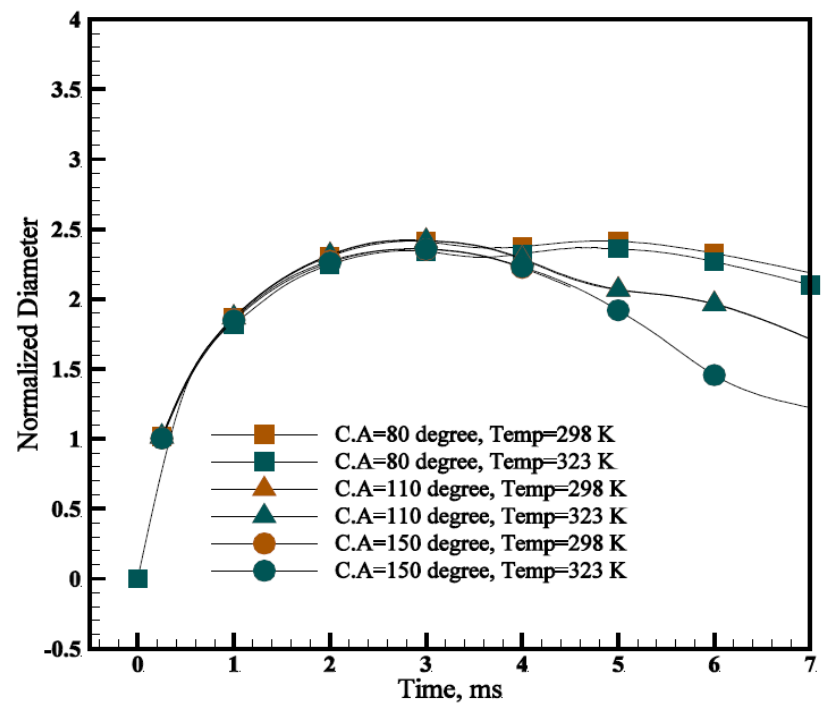

Figure. 12. Temperature effects on non-Newtonian droplets behavior on surfaces with different contact angles 


\section{CONCLUSIONS}

The hitting effects of Newtonian and non-Newtonian drops on hydrophobic surface have been studied at different Weber numbers along the present work. In this section, the general obtaining results is described case by case:

1. Simulation of droplet hitting with a hydrophobic and superhydrophobic surface has contained good results, and schematic and graphing results are acceptable.

2. At first, wet diameter increases and then decreases, also we can see reduction of this changing rate, by decreasing the velocity of the drop hitting on surface.

3. The proposed method is general for the similar tasks so that this method can be used for any Newtonian and non-Newtonian fluids.

4. The velocity at the drop peak is lower for larger Weber numbers in droplet hitting on surface at a certain time.

5. Temperature effects on hydrophobic surfaces is ignorable.

\section{REFERENCES}

[1] Watanabe, K., Y. Udagawa, and H. Udagawa, Drag reduction of Newtonian fluid in a circular pipe with a highly water-repellent wall. Journal of Fluid Mechanics, 1999. 381: p. 225-238.

[2] Tretheway, D.C. and C.D. Meinhart, A generating mechanism for apparent fluid slip in hydrophobic microchannels. Physics of Fluids (1994-present), 2004. 16(5): p. 1509-1515.

[3] Min, T. and J. Kim, Effects of hydrophobic surface on skin-friction drag. Physics of Fluids (1994-present), 2004. 16(7): p. L55-L58.

[4] Choi, C.-H., K.J.A. Westin, and K.S. Breuer, Apparent slip flows in hydrophilic and hydrophobic microchannels. Physics of Fluids (1994-present), 2003. 15(10): p. 2897-2902.

[5] Ou, J., B. Perot, and J.P. Rothstein, Laminar drag reduction in microchannels using ultrahydrophobic surfaces. Physics of Fluids (1994-present), 2004. 16(12): p. 4635-4643.

[6] Yong-Sheng, Y. and W. Qing-Ding, Experimental study on physical mechanism of drag reduction of hydrophobic materials in laminar flow. Chinese physics letters, 2006. 23(6): p. 1634.

[7] Daniello, R.J., N.E. Waterhouse, and J.P. Rothstein, Drag reduction in turbulent flows over superhydrophobic surfaces. Physics of Fluids (1994-present), 2009. 21(8): p. 085103.

[8] Pittoni, P.G., Y.-C. Lin, and S.-Y. Lin, The impalement of water drops impinging onto hydrophobic/superhydrophobic graphite surfaces: the role of dynamic pressure, hammer pressure and liquid penetration time. Applied Surface Science, 2014. 301: p. 515-524. 